Available online at http://jnfa.mathres.org
MATHES Journal of Nonlinear Functional Analysis
A

\title{
A MODIFIED POPOV'S SUBGRADIENT EXTRAGRADIENT METHOD FOR VARIATIONAL INEQUALITIES IN BANACH SPACES
}

\author{
PONGSAKORN SUNTHRAYUTH ${ }^{1, *}$, HABIB UR REHMAN ${ }^{2}$, POOM KUMAM $^{2}$ \\ ${ }^{1}$ Department of Mathematics and Computer Science, Faculty of Science and Technology, \\ Rajamangala University of Technology Thanyaburi (RMUTT), \\ 39 Rangsit-Nakhonnayok Rd., Klong 6, Thanyaburi, Pathumthani, 12110, Thailand \\ ${ }^{2}$ KMUTT Fixed Point Research Laboratory, KMUTT-Fixed Point Theory and Applications Research Group, \\ SCL 802 Fixed Point Laboratory, Department of Mathematics, Faculty of Science, \\ King Mongkut's University of Technology Thonburi (KMUTT), Thrung Khru, Bangkok 10140, Thailand
}

\begin{abstract}
In this paper, we propose a new modification of Popov's subgradient extragradient method for solving the variational inequality problem involving pseudo-monotone and Lipschitz-continuous mappings in the framework of Banach spaces. The weak convergence theorem of the proposed method is established without the knowledge of the Lipschitz constant of the Lipschitz continuous mapping. Finally, we provide several numerical experiments of the proposed method including comparisons with other related methods. Our result generalizes and extends many related results in the literature from Hilbert spaces to Banach spaces.
\end{abstract}

Keywords. Popov's method; Variational inequality problem; Pseudo-monotone mapping; Banach space.

\section{INTRODUCTION}

Let $E$ be a real Banach space with norm $\|\cdot\|$ and let $E^{*}$ be the dual space of $E$. We denote by $\langle x, f\rangle$ the value of $f \in E^{*}$ at $x \in E$, that is, $\langle x, f\rangle=f(x)$. Let $C$ be a nonempty, closed and convex subset of $E$ and let $A: C \rightarrow E^{*}$ be a mapping. The variational inequality problem (VIP) is to find an element $z \in C$ such that

$$
\langle x-z, A z\rangle \geq 0, \quad \forall x \in C .
$$

The solution set of the VIP is denoted by $\operatorname{VI}(C, A)$. The VIP has been studied widely in many real-world problems, such as, artificial intelligence, computer science, control engineering, management science and operations research, and differential equations, fluid flow through

\footnotetext{
${ }^{*}$ Corresponding author.

E-mail addresses: pongsakorn_su@rmutt.ac.th (P. Sunthrayuth), hrehman.hed@gmail.com (H.U. Rehman), poom.kum@kmutt.ac.th (P. Kumam)

Received October 8, 2020; Accepted March 9, 2021.
}

(C)2021 Journal of Nonlinear Functional Analysis 
porous media, contact problems in elasticity, transportation problems and economics equilibrium; see, e.g. $[1,2,3,4,5,6,7]$ and the references therein.

A simple method for solving VIP in a real Hilbert space $H$ is known as the projected gradient method, which is defined by the following scheme:

$$
x_{n+1}=P_{C}\left(x_{n}-\lambda A x_{n}\right),
$$

where $P_{C}$ is the projection operator onto the convex and closed subset $C$ of $H$ and $\lambda>0$ is a suitable stepsize. This method converges weakly to a solution of VIP under the following Assumption $(a 1)$ or $(a 2)$

(a1) $A$ is strongly monotone and Lipschitz continuous, and $\lambda \in\left(0, \frac{2 \gamma}{L^{2}}\right)$;

(a2) $A$ is inverse strongly monotone and $\lambda \in(0,2 \alpha)$,

where $\gamma>0$ and $L>0$ are strongly monotone and Lipschitz constants, respectively, and $\alpha>0$ is the inverse strongly monotone constant of $A$.

We remark that Assumptions $(a 1)$ and $(a 2)$ are strong. Without the strong monotonicity, this method may diverges (see, e.g., [8]). In order to improve this drawback, Korpelevich [9] introduced the so-called extragradient method for solving the VIP in a finite-dimensional Euclidean space $\mathbb{R}^{m}$ as follows:

$$
\left\{\begin{array}{l}
y_{n}=P_{C}\left(x_{n}-\lambda A x_{n}\right), \\
x_{n+1}=P_{C}\left(x_{n}-\lambda A y_{n}\right),
\end{array}\right.
$$

where $C \subset \mathbb{R}^{m}$ is a nonempty, closed and convex set, $A: C \rightarrow \mathbb{R}^{m}$ is monotone and $L$-Lipschitz continuous, and $\lambda \in\left(0, \frac{1}{L}\right)$. It was proved that if $V I(C, A)$ is nonempty, then the sequences $\left\{x_{n}\right\}$ generated by (1.3) converges to an element in $\operatorname{VI}(C, A)$. The idea of the extragradient method was successfully generalized and extended not only in Euclidean spaces but also in Hilbert and Banach spaces. In recent years, the extragradient method was further studied intensively and it has been extended in various ways by many authors; see, e.g., $[10,11,12,13,14,15,16]$ and the references therein. Note that the extragradient method was based on a double-projection method onto the feasible set $C$. It needs to compute two projections onto $C$ in each iteration step. In fact, in some cases, the structure of the set $C$ is not explicit or complicated. As a result, the projection onto $C$ might be difficult to compute. Moreover, in each iteration step of the extragradient method, one has to compute two values of the mapping $A$ at points $x_{n}$ and $y_{n}$.

To deal with the improvement of the extragradient method (1.3), Popov [17] proposed the socalled Popov's extragradient method, which only requires to compute one value of the mapping $A$ at one point $y_{n}$ per iteration. The Popov's extragradient method is of the form:

$$
\left\{\begin{array}{l}
x_{n+1}=P_{C}\left(x_{n}-\lambda A y_{n}\right), \\
y_{n+1}=P_{C}\left(x_{n+1}-\lambda A y_{n}\right),
\end{array}\right.
$$

where $\lambda \in\left(0, \frac{1}{3 L}\right)$. In [17], the convergence of this method was proved in a finite dimensional Euclidean space.

In [18], Censor, Gibali and Reich proposed the so-called subgradient extragradient method for solving the VIP in a real Hilbert space $H$. They replaced the second projection onto $C$ of the extragradient method (1.3) by a projection onto a half-space, which is easier to compute. The 
subgradient extragradient method is of the form:

$$
\left\{\begin{array}{l}
y_{n}=P_{C}\left(x_{n}-\lambda A x_{n}\right), \\
T_{n}=\left\{x \in H:\left\langle x_{n}-\lambda_{n} A x_{n}-y_{n}, x-y_{n}\right\rangle \leq 0\right\}, \\
x_{n+1}=P_{T_{n}}\left(x_{n}-\lambda A y_{n}\right),
\end{array}\right.
$$

where $C \subset H$ is a nonempty, closed and convex set, $A: C \rightarrow H$ is monotone and $L$-Lipschitz continuous, and $\lambda \in\left(0, \frac{1}{L}\right)$. However, this method was still required to compute the value of the mapping $A$ at two different points in each iteration step.

Recently, Malitsky and Semenov [19] combined the advantages of Popov's extragradient method (1.4) and subgradient extragradient method (1.5). They proposed the so-called Popov's subgradient extragradient method for solving VIP in a real Hilbert space $H$ as follows:

$$
\left\{\begin{array}{l}
y_{n+1}=P_{C}\left(x_{n+1}-\lambda A y_{n}\right), \\
T_{n}=\left\{x \in H:\left\langle x_{n}-\lambda_{n} A y_{n-1}-y_{n}, x-y_{n}\right\rangle \leq 0\right\}, \\
x_{n+1}=P_{T_{n}}\left(x_{n}-\lambda A y_{n}\right),
\end{array}\right.
$$

where $\lambda \in\left(0, \frac{1}{3 L}\right)$. It was proved that the sequence $\left\{x_{n}\right\}$ generated by (1.6) converges weakly to a solution of the VIP provided that $\operatorname{VI}(C, A)$ is nonempty. We remark that this method is not only requires to compute one projection onto the feasible set $C$ but it also compute one value of the mapping $A$ in each iteration step. In view of this, the Popov's subgradient extragradient method has received great attention in various ways; see, e.g., [20, 21, 22] and the references therein. However, the Popov's subgradient extragradient method (1.6) requires to know the Lipschitz constant or at least to know some estimation of it.

Motivated and inspired by the previous works, in this paper, we extend the Popov's subgradient extragradient method (1.6) from Hilbert spaces to Banach spaces, which are 2-uniformly convex and uniformly smooth. We prove the weak convergence result of the proposed algorithm to a solution of th VIP when $A$ is pseudo-monotone and Lipschitz continuous. The advantage of our algorithm is that the stepsize does not requires to know the Lipschitz constant of the Lipschitz continuous mapping.

The outline of this paper is organized as follows: In Section 2, some preliminaries and facts are presented. In Section 3, we prove the weak convergence result of the proposed algorithm. Finally, in Section 4, we perform several numerical experiments to show the efficiency and advantages of the proposed algorithm. The result in this paper generalizes and extends many known results in the literature.

\section{PRELIMINARIES}

Throughout this paper, we denote the set of real numbers and the set of positive integers by $\mathbb{R}$ and $\mathbb{N}$, respectively. Let $E$ be a real Banach space and $E^{*}$ the dual space of $E$. For a sequence $\left\{x_{n}\right\} \subset E$, we denote $x_{n} \rightarrow x$ and $x_{n} \rightarrow x$ by the strong convergence and the weak convergence of $\left\{x_{n}\right\}$ to $x$, respectively. Let $U=\{x \in E:\|x\|=1\}$ be the unit sphere of $E$.

Definition 2.1. [23, 24, 25] Let $E$ be a Banach space.

(1) The modulus of convexity $\delta_{E}:[0,2] \rightarrow[0,1]$ is defined by

$$
\delta_{E}(\varepsilon)=\inf \left\{1-\frac{\|x+y\|}{2}: x, y \in U,\|x-y\| \geq \varepsilon\right\} .
$$


(2) The modulus of smoothness $\rho_{E}:[0, \infty) \rightarrow[0, \infty)$ is defined by

$$
\rho_{E}(t)=\sup \left\{\frac{\|x+t y\|+\|x-t y\|}{2}-1: x, y \in U\right\} .
$$

Definition 2.2. $[23,24,25]$ A Banach space $E$ is said to be:

(1) strictly convex if $\frac{\|x+y\|}{2}<1$ for all $x, y \in U$ and $x \neq y$;

(2) smooth if $\lim _{t \rightarrow 0} \frac{\|x+t y\|-\|x\|}{t}$ exists for all $x, y \in U$;

(3) uniformly convex if $\delta_{E}(\varepsilon)>0$ for alle $\in(0,2]$;

(4) uniformly smooth if $\lim _{t \rightarrow 0} \frac{\rho_{E}(t)}{t}=0$;

(5) p-uniformly convex if there exist $c>0$ and $p \geq 2$ such that $\delta_{E}(\varepsilon) \geq c \varepsilon^{p}$ for all $\varepsilon \in(0,2]$;

(6) $q$-uniformly smooth if there exist $\kappa>0$ and $1<q \leq 2$ such that $\rho_{E}(t) \leq \kappa t^{q}$ for all $t>0$.

Lemma 2.3. [23, 24, 25] Let $E$ be a Banach space. Let $1<q \leq 2<p<\infty$ with $\frac{1}{p}+\frac{1}{q}=1$. Then the following statements hold:

(i) If $E$ is p-uniformly convex (q-uniformly smooth), then $E$ is uniformly convex (uniformly smooth.

(ii) $E$ is p-uniformly convex (q-uniformly smooth) if and only if its dual $E^{*}$ is q-uniformly smooth (p-uniformly convex).

(iii) If $E$ is uniformly convex (uniformly smooth), then $E$ is strictly convex and reflexive (reflexive and smooth).

Remark 2.4. Typical examples of both uniformly convex and uniformly smooth Banach spaces are $L_{p}$ and $\ell_{p}$, where $p>1$. More precisely, $L_{p}$ and $\ell_{p}$ are $\max \{p, 2\}$-uniformly convex and $\min \{p, 2\}$-uniformly smooth, while Hilbert spaces are 2-uniformly convex and 2-uniformly smooth (see [26] for more details).

Definition 2.5. [23, 24, 25] Let $E$ be a Banach space.

(1) The duality mapping $J: E \rightarrow 2^{E^{*}}$ is defined by $J x=\left\{f \in E^{*}:\langle x, f\rangle=\|x\|^{2}=\|f\|^{2}\right\}$ for all $x \in E$.

(2) The duality mapping $J$ from $E$ into $E^{*}$ is said to be weakly sequentially continuous if for any sequence $\left\{x_{n}\right\} \subset E$ such that $x_{n} \rightarrow x$ implies that $J x_{n} \rightarrow^{*} J x$.

Lemma 2.6. $[23,24,25]$ Let $E$ be a Banach space and let $J$ be the duality mapping on $E$. The following facts are well-known:

(i) If E is smooth, then J is single-valued and monotone, that is,

$$
\langle x-y, J x-J y\rangle \geq 0, \forall x, y \in E .
$$

Further, if $E$ is strictly convex and $\langle x-y, J x-J y\rangle=0$, then $x=y$.

(ii) If $E$ is strictly convex, then $J$ is one-to-one.

(iii) If $E$ is uniformly smooth, then $J$ is norm-to-norm uniformly continuous on bounded subsets of $E$.

(iv) If $E$ is reflexive, smooth and strictly convex, then $J^{-1}$ is single-valued, one-to-one, surjective and it is the duality mapping from $E^{*}$ into $E$.

(v) If $E$ is a Hilbert space, then $J$ is the identity mapping.

Definition 2.7. Let $C$ be a nonempty subset of a Banach space $E$. A mapping $A: C \rightarrow E^{*}$ is said to be: 
(1) monotone if

$$
\langle x-y, A x-A y\rangle \geq 0, \forall x, y \in C
$$

(2) pseudo-monotone if

$$
\langle y-x, A x\rangle \geq 0 \Longrightarrow\langle y-x, A y\rangle \geq 0, \forall x, y \in C ;
$$

(3) Lipschitz continuous if there exists a constant $L>0$ such that

$$
\|A x-A y\| \leq L\|x-y\|, \forall x, y \in C ;
$$

(4) hemicontinuous if for each $x, y \in C$, the mapping $f:[0,1] \rightarrow E^{*}$ defined by

$$
f(t)=A(t x+(1-t) y)
$$

is continuous with respect to the weak* topology of $E^{*}$.

Remark 2.8. Every monotone mapping is a pseudo-monotone mapping but converse is not true in general. The example of a pseudo-monotone mapping but is not monotone can be found in [27].

Definition 2.9. [28] Let $E$ be a smooth Banach space. The Lyapunov function $\phi: E \times E \rightarrow \mathbb{R}$ is defined by

$$
\phi(x, y)=\|x\|^{2}-2\langle x, J y\rangle+\|y\|^{2}, \forall x, y \in E .
$$

If $E$ is a Hilbert space, then $\phi(x, y)=\|x-y\|^{2}$ for all $x, y \in E$. From the definition of $\phi$, it is clear that

$$
(\|x\|-\|y\|)^{2} \leq \phi(x, y) \leq(\|x\|+\|y\|)^{2}, \forall x, y \in E .
$$

From (2.1), we can see that $\phi(x, y) \geq 0$ and if $E$ is additionally assumed to be strictly convex, then

$$
\phi(x, y)=0 \Longleftrightarrow x=y .
$$

Furthermore, the function $\phi$ has the following two important properties:

$$
\phi(x, y)+\phi(y, x)=2\langle x-y, J x-J y\rangle, \forall x, y \in E
$$

and

$$
\phi(x, y)=\phi(x, z)+\phi(z, y)+2\langle x-z, J z-J y\rangle, \forall x, y, z \in E .
$$

Following [28], we have the functional $V: E \times E^{*} \rightarrow \mathbb{R}$, which is defined by

$$
V\left(x, x^{*}\right)=\|x\|^{2}-2\left\langle x, x^{*}\right\rangle+\left\|x^{*}\right\|^{2}, \forall x \in E, x^{*} \in E^{*} .
$$

Then $V$ is nonnegative and $V\left(x, x^{*}\right)=\phi\left(x, J^{-1} x^{*}\right)$ for all $x \in E$ and $x^{*} \in E^{*}$.

Definition 2.10. [28] Let $E$ be a reflexive, strictly convex and smooth Banach space. Let $C$ be a nonempty, closed convex subset of $E$. The generalized projection mapping is a mapping $\Pi_{C}: E \rightarrow C$ that assigns an arbitrary element $x \in E$ to the minimum element of the function $\phi(y, x)$, that is, $\Pi_{C} x=z$, where $z$ is the solution to the following minimization problem:

$$
\phi(z, x)=\min _{y \in C} \phi(y, x) .
$$

Remark 2.11. If $E$ is a Hilbert space, then $\Pi_{C}$ is coincident with the metric projection, denoted by $P_{C}$. 
Lemma 2.12. [28] Let $E$ be a reflexive, strictly convex and smooth Banach space and let $C$ be a nonempty, closed and convex subset of $E$. Let $x \in E$ and $z \in C$. Then the following statements hold:

(i) $z=\Pi_{C}(x)$ if and only if $\langle y-z, J z-J x\rangle \geq 0, \forall y \in C$.

(ii) $\phi\left(y, \Pi_{C}(x)\right)+\phi\left(\Pi_{C}(x), x\right) \leq \phi(y, x), \forall y \in C$.

Lemma 2.13. [29] Let E be a 2-uniformly convex Banach space. Then there exists a constant $\delta \geq 1$ such that

$$
\frac{1}{\delta}\|x-y\|^{2} \leq \phi(x, y), \forall x, y \in E .
$$

Lemma 2.14. [10] Let $C$ be a nonempty, closed and convex subset of a reflexive Banach space $E$. Let $A: C \rightarrow E^{*}$ be a pseudo-monotone and hemicontinuous operator. Then

(i) $z$ is a solution of VIP (1.1) if and only if $\langle x-z, A x\rangle \geq 0, \forall x \in C$.

(ii) $\operatorname{VI}(C, A)$ is closed and convex.

The following fact can be found in [30, Lemma 3.1].

Lemma 2.15. For any $a, b \in \mathbb{R}$ and $\varepsilon>0$, the following inequality holds

$$
2 a b \leq \frac{a^{2}}{\varepsilon}+\varepsilon b^{2}
$$

Proof. Since $0 \leq\left(\frac{1}{\sqrt{\varepsilon}} a-\sqrt{\varepsilon} b\right)^{2}=\frac{a^{2}}{\varepsilon}-2 a b+\varepsilon b^{2}$, we have $2 a b \leq \frac{a^{2}}{\varepsilon}+\varepsilon b^{2}$.

Lemma 2.16. Let $\left\{\mathscr{A}_{n}\right\}$ and $\left\{\mathscr{B}_{n}\right\}$ be two nonnegative real sequences such that

$$
\mathscr{A}_{n+1} \leq \mathscr{A}_{n}-\mathscr{B}_{n}, \forall n \geq 1
$$

Then $\lim _{n \rightarrow \infty} \mathscr{A}_{n}$ exists and $\sum_{n=1}^{\infty} \mathscr{B}_{n}<\infty$.

The following lemma will be needed in the proof of the main result.

Lemma 2.17. Let $C$ be a nonempty, closed and convex subset of a real 2-uniformly convex Banach space E, which is also uniformly smooth. Let $\left\{x_{n}\right\}$ be a sequence in E. Suppose that the following two conditions hold:

(i) $\lim _{n \rightarrow \infty} \phi\left(u, x_{n}\right)$ exists for each $u \in C$;

(ii) every sequential weak limit point of $\left\{x_{n}\right\}$ belongs to $C$.

Suppose, in addition, that $J$ is weakly sequentially continuous on E. Then $\left\{x_{n}\right\}$ converges weakly to some element in $C$.

Proof. Since $\lim _{n \rightarrow \infty} \phi\left(u, x_{n}\right)$ exists, we have that $\left\{\phi\left(u, x_{n}\right)\right\}$ is bounded. Applying Lemma 2.13, we obtain that $\left\{x_{n}\right\}$ is bounded. By the reflexivity and the boundedness of $\left\{x_{n}\right\}$, we can suppose that there are two subsequences $\left\{x_{n_{k}}\right\}$ and $\left\{x_{m_{k}}\right\}$ of $\left\{x_{n}\right\}$ such that $x_{n_{k}} \rightarrow u$ and $x_{m_{k}} \rightarrow v$ for some $u, v \in C$. From this, we see that $\lim _{n \rightarrow \infty}\left(\phi\left(u, x_{n}\right)-\phi\left(v, x_{n}\right)\right)$ exists. From the definition of $\phi$, we have

$$
\begin{aligned}
\phi\left(u, x_{n}\right)-\phi\left(v, x_{n}\right) & =\|u\|^{2}-2\left\langle u, J x_{n}\right\rangle+\left\|x_{n}\right\|^{2}-\left(\|v\|^{2}-2\left\langle v, J x_{n}\right\rangle+\left\|x_{n}\right\|^{2}\right) \\
& =\|u\|^{2}-\|v\|^{2}-2\left\langle u-v, J x_{n}\right\rangle
\end{aligned}
$$


and then

$$
\|u\|^{2}-\|v\|^{2}-2 \lim _{k \rightarrow \infty}\left\langle u-v, J x_{n_{k}}\right\rangle=\|u\|^{2}-\|v\|^{2}-2 \lim _{k \rightarrow \infty}\left\langle u-v, J x_{m_{k}}\right\rangle .
$$

Since $J$ is weakly sequentially continuous, we have $\langle u-v, J u\rangle=\langle u-v, J v\rangle$, that is, $\langle u-v, J u-$ $J v\rangle=0$. By the strict convexity of $E$, we obtain $u=v$. This completes the proof.

\section{MAin Result}

In this section, we propose a modification of Popov's subgradient extragradient method for solving pseudo-monotone variational inequalities in Banach spaces. In order to prove convergence of the proposed method, we need the following assumptions:

(A1) The set $C$ is a nonempty, closed and convex subset of a real 2-uniformly convex Banach space $E$, which is also uniformly smooth.

(A2) The mapping $A: E \rightarrow E^{*}$ is pseudo-monotone and $L$-Lipschitz continuous.

(A3) The duality mapping $J$ is weakly sequentially continuous on $E$.

(A4) The solution set of the VIP is nonempty, that is, $V I(C, A) \neq \emptyset$.

\section{Algorithm 1 Modified Popov's subgradient extragradient algorithm}

Step 0: Give $\lambda_{0}, \lambda_{1}>0$ and $\mu \in\left(0, \frac{\sqrt{2}-1}{\delta}\right)$, where $\delta$ is a constant given by Lemma 2.13. Let $x_{0}, y_{0} \in C$ be arbitrary.

Step 1: Compute

$$
\left\{\begin{array}{l}
x_{1}=\Pi_{C} J^{-1}\left(J x_{0}-\lambda_{0} A y_{0}\right) \\
y_{1}=\Pi_{C} J^{-1}\left(J x_{1}-\lambda_{1} A y_{0}\right)
\end{array}\right.
$$

Step 2: Given the current iterate $x_{n}, y_{n}$ and $y_{n-1}$, calculate $x_{n+1}$ as follows:

$$
x_{n+1}=\Pi_{T_{n}} J^{-1}\left(J x_{n}-\lambda_{n} A y_{n}\right),
$$

where

$$
T_{n}=\left\{x \in E:\left\langle x-y_{n}, J x_{n}-\lambda_{n} A y_{n-1}-J y_{n}\right\rangle \leq 0\right\} .
$$

Step 3: Compute

$$
y_{n+1}=\Pi_{C} J^{-1}\left(J x_{n+1}-\lambda_{n+1} A y_{n}\right),
$$

where

$$
\lambda_{n+1}= \begin{cases}\min \left\{\mu \frac{\sqrt{2}\left\|x_{n+1}-y_{n}\right\|^{2}+\frac{1}{\sqrt{2}}\left\|y_{n}-y_{n-1}\right\|^{2}}{2\left\langle x_{n+1}-y_{n}, A y_{n-1}-A y_{n}\right\rangle}, \lambda_{n}\right\} & \text { if }\left\langle x_{n+1}-y_{n}, A y_{n-1}-A y_{n}\right\rangle>0 \\ \lambda_{n} & \text { otherwise. }\end{cases}
$$

If $x_{n+1}=x_{n}$ and $y_{n-1}=y_{n}\left(\right.$ or $\left.x_{n+1}=y_{n}=y_{n+1}\right)$, then stop and $y_{n}$ is a solution of the VIP. Otherwise, go to Step 1.

Lemma 3.1. Let $\left\{\lambda_{n}\right\}$ be a sequence generated by (3.5). Then $\left\{\lambda_{n}\right\}$ is nonincreasing and

$$
\lim _{n \rightarrow \infty} \lambda_{n}=\lambda \geq \min \left\{\frac{\mu}{L}, \lambda_{1}\right\} .
$$


Moreover,

$$
2\left\langle x_{n+1}-y_{n}, A y_{n-1}-A y_{n}\right\rangle \leq \frac{\mu}{\lambda_{n+1}}\left(\sqrt{2}\left\|x_{n+1}-y_{n}\right\|^{2}+\frac{1}{\sqrt{2}}\left\|y_{n}-y_{n-1}\right\|^{2}\right), \forall n \geq 1 .
$$

Proof. It is obvious from (3.5) that $\lambda_{n+1} \leq \lambda_{n}$ for all $n \geq 1$. For the case $\left\langle x_{n+1}-y_{n}, A y_{n}-\right.$ $\left.A y_{n-1}\right\rangle>0$, Lemma 2.15 and the $L$-Lipschitz continuity of $A$, we have

$$
\mu \frac{\sqrt{2}\left\|x_{n+1}-y_{n}\right\|^{2}+\frac{1}{\sqrt{2}}\left\|y_{n}-y_{n-1}\right\|^{2}}{2\left\langle x_{n+1}-y_{n}, A y_{n-1}-A y_{n}\right\rangle} \geq \frac{2 \mu\left\|x_{n+1}-y_{n}\right\|\left\|y_{n}-y_{n-1}\right\|}{2 L\left\|x_{n+1}-y_{n}\right\|\left\|y_{n}-y_{n-1}\right\|}=\frac{\mu}{L} .
$$

Clearly,

$$
\lambda_{n+1} \geq \min \left\{\frac{\mu}{L}, \lambda_{n}\right\}
$$

By induction, we immediately obtain that $\left\{\lambda_{n}\right\}$ is bounded from below by $\min \left\{\frac{\mu}{L}, \lambda_{1}\right\}$. Thus there exists $\lambda:=\lim _{n \rightarrow \infty} \lambda_{n} \geq \min \left\{\frac{\mu}{L}, \lambda_{1}\right\}$.

On the other hand, from the definition of $\lambda_{n}$, we have

$$
\begin{aligned}
\lambda_{n+1} & =\min \left\{\mu \frac{\sqrt{2}\left\|x_{n+1}-y_{n}\right\|^{2}+\frac{1}{\sqrt{2}}\left\|y_{n}-y_{n-1}\right\|^{2}}{2\left\langle x_{n+1}-y_{n}, A y_{n-1}-A y_{n}\right\rangle}, \lambda_{n}\right\} \\
& \leq \mu \frac{\sqrt{2}\left\|x_{n+1}-y_{n}\right\|^{2}+\frac{1}{\sqrt{2}}\left\|y_{n}-y_{n-1}\right\|^{2}}{2\left\langle x_{n+1}-y_{n}, A y_{n-1}-A y_{n}\right\rangle} .
\end{aligned}
$$

This implies that

$$
2\left\langle x_{n+1}-y_{n}, A y_{n-1}-A y_{n}\right\rangle \leq \frac{\mu}{\lambda_{n+1}}\left(\sqrt{2}\left\|x_{n+1}-y_{n}\right\|^{2}+\frac{1}{\sqrt{2}}\left\|y_{n}-y_{n-1}\right\|^{2}\right), \forall n \geq 1 .
$$

Lemma 3.2. Let $\left\{x_{n}\right\}$ be a sequence generated by Algorithm 1. If $x_{n+1}=x_{n}$ and $y_{n-1}=y_{n}$, then $y_{n}$ is a solution of the VIP.

Proof. If $x_{n+1}=x_{n}$, then $x_{n}=\Pi_{T_{n}} J^{-1}\left(J x_{n}-\lambda_{n} A y_{n}\right)$. By Lemma $2.12(i)$, we have

$$
\begin{aligned}
\left\langle x-x_{n}, J x_{n}-J \circ J^{-1}\left(J x_{n}-\lambda_{n} A y_{n}\right)\right\rangle & =\left\langle x-x_{n}, J x_{n}-J x_{n}+\lambda_{n} A y_{n}\right\rangle \\
& =\lambda_{n}\left\langle x-x_{n}, A y_{n}\right\rangle \geq 0, \forall x \in T_{n} .
\end{aligned}
$$

This implies that $\left\langle x-y_{n}, A y_{n}\right\rangle \geq\left\langle x_{n}-y_{n}, A y_{n}\right\rangle, \forall x \in T_{n}$. It is easy to see that $C \subset T_{n}$, and

$$
\left\langle x-y_{n}, A y_{n}\right\rangle \geq\left\langle x_{n}-y_{n}, A y_{n}\right\rangle, \forall x \in C .
$$

On the other hand, by the definition of $T_{n}$, we have

$$
\left\langle x-y_{n}, J x_{n}-\lambda_{n} A y_{n-1}-J y_{n}\right\rangle \leq 0, \forall x \in T_{n} .
$$

Since $x_{n}=x_{n+1}$, we have $x_{n} \in T_{n}$. If $y_{n-1}=y_{n}$, then $\left\langle x_{n}-y_{n}, J x_{n}-\lambda_{n} A y_{n}-J y_{n}\right\rangle \leq 0$. From (2.3), we see that

$$
\left\langle x_{n}-y_{n}, A y_{n}\right\rangle \geq\left\langle x_{n}-y_{n}, J x_{n}-J y_{n}\right\rangle \geq \frac{1}{2} \phi\left(x_{n}, y_{n}\right) \geq 0 .
$$

Combining (3.6) and (3.7), we thus get $\left\langle x-y_{n}, A y_{n}\right\rangle \geq 0, \forall x \in C$. Hence, $y_{n}$ is a solution of the VIP. 
Lemma 3.3. Assume that Assumptions $(A 1)-(A 4)$ hold. Let $\left\{x_{n}\right\}$ be a sequence generated by Algorithm 1. For each $n \geq 1$, we have

$$
\mathscr{A}_{n+1} \leq \mathscr{A}_{n}-\left(1-(\sqrt{2}+1) \theta_{n}\right) \phi\left(y_{n}, x_{n}\right)-\left(1-\sqrt{2} \theta_{n}-\theta_{n+1}\right) \phi\left(x_{n+1}, y_{n}\right), \forall p \in V I(C, A),
$$

where $\mathscr{A}_{n}=\phi\left(p, x_{n}\right)+\theta_{n} \phi\left(x_{n}, y_{n-1}\right)$ and $\theta_{n}=\frac{\mu \delta \lambda_{n}}{\lambda_{n+1}}$.

Proof. Let $p \in V I(C, A)$. By Lemma $2.12(i i)$, we have

$$
\begin{aligned}
\phi & \left(p, x_{n+1}\right) \\
= & \phi\left(p, \Pi_{T_{n}} J^{-1}\left(J x_{n}-\lambda_{n} A y_{n}\right)\right) \\
\leq & \phi\left(p, J^{-1}\left(J x_{n}-\lambda_{n} A y_{n}\right)\right)-\phi\left(x_{n+1}, J^{-1}\left(J x_{n}-\lambda_{n} A y_{n}\right)\right) \\
= & V\left(p, J x_{n}-\lambda_{n} A y_{n}\right)-V\left(x_{n+1}, J x_{n}-\lambda_{n} A y_{n}\right) \\
= & \|p\|^{2}-2\left\langle p, J x_{n}\right\rangle+2 \lambda_{n}\left\langle p, A y_{n}\right\rangle+\left\|J x_{n}-\lambda_{n} A y_{n}\right\|^{2}-\left\|x_{n+1}\right\|^{2} \\
& +2\left\langle x_{n+1}, J x_{n}\right\rangle-2 \lambda_{n}\left\langle x_{n+1}, A y_{n}\right\rangle-\left\|J x_{n}-\lambda_{n} A y_{n}\right\|^{2} \\
= & \|p\|^{2}-2\left\langle p, J x_{n}\right\rangle+\left\|x_{n}\right\|^{2}-\left(\left\|x_{n+1}\right\|^{2}-2\left\langle x_{n+1}, J x_{n}\right\rangle+\left\|x_{n}\right\|^{2}\right)-2 \lambda_{n}\left\langle x_{n+1}-p, A y_{n}\right\rangle \\
= & \phi\left(p, x_{n}\right)-\phi\left(x_{n+1}, x_{n}\right)-2 \lambda_{n}\left\langle x_{n+1}-p, A y_{n}\right\rangle \\
= & \phi\left(p, x_{n}\right)-\phi\left(x_{n+1}, x_{n}\right)-2 \lambda_{n}\left\langle x_{n+1}-y_{n}, A y_{n}\right\rangle-2 \lambda_{n}\left\langle y_{n}-p, A y_{n}\right\rangle .
\end{aligned}
$$

Since $y_{n} \in C$, we have $\left\langle y_{n}-p, A p\right\rangle \geq 0$. By the pseudo-monotonicity of $A$, we have $\left\langle y_{n}-\right.$ $\left.p, A y_{n}\right\rangle \geq 0$. It follows from (3.8) that

$$
\phi\left(p, x_{n+1}\right) \leq \phi\left(p, x_{n}\right)-\phi\left(x_{n+1}, x_{n}\right)-2 \lambda_{n}\left\langle x_{n+1}-y_{n}, A y_{n}\right\rangle
$$

From (2.3), we see that

$$
\phi\left(x_{n+1}, x_{n}\right)=\phi\left(x_{n+1}, y_{n}\right)+\phi\left(y_{n}, x_{n}\right)+2\left\langle x_{n+1}-y_{n}, J y_{n}-J x_{n}\right\rangle .
$$

Substituting (3.10) and (3.9), we have

$$
\begin{aligned}
\phi\left(p, x_{n+1}\right) \leq & \phi\left(p, x_{n}\right)-\phi\left(x_{n+1}, y_{n}\right)-\phi\left(y_{n}, x_{n}\right)-2\left\langle x_{n+1}-y_{n}, J y_{n}-J x_{n}\right\rangle-2 \lambda_{n}\left\langle x_{n+1}-y_{n}, A y_{n}\right\rangle \\
= & \phi\left(p, x_{n}\right)-\phi\left(x_{n+1}, y_{n}\right)-\phi\left(y_{n}, x_{n}\right)+2 \lambda_{n}\left\langle x_{n+1}-y_{n}, A y_{n-1}-A y_{n}\right\rangle \\
& +2\left\langle x_{n+1}-y_{n}, J x_{n}-\lambda_{n} A y_{n-1}-J y_{n}\right\rangle .
\end{aligned}
$$

Since $x_{n+1} \in T_{n}$, we have $\left\langle x_{n+1}-y_{n}, J x_{n}-\lambda_{n} A y_{n-1}-J y_{n}\right\rangle \leq 0$. This implies that

$$
\phi\left(p, x_{n+1}\right) \leq \phi\left(p, x_{n}\right)-\phi\left(x_{n+1}, y_{n}\right)-\phi\left(y_{n}, x_{n}\right)+2 \lambda_{n}\left\langle x_{n+1}-y_{n}, A y_{n-1}-A y_{n}\right\rangle
$$

From Lemma 3.1, we see that

$$
2 \lambda_{n}\left\langle x_{n+1}-y_{n}, A y_{n-1}-A y_{n}\right\rangle \leq \mu \frac{\lambda_{n}}{\lambda_{n+1}}\left(\sqrt{2}\left\|x_{n+1}-y_{n}\right\|^{2}+\frac{1}{\sqrt{2}}\left\|y_{n-1}-y_{n}\right\|^{2}\right) .
$$


Now, we estimate $\left\|y_{n-1}-y_{n}\right\|^{2}$. Observe that

$$
\begin{aligned}
\left\|y_{n-1}-y_{n}\right\|^{2} & \leq\left(\left\|y_{n}-x_{n}\right\|+\left\|x_{n}-y_{n-1}\right\|\right)^{2} \\
& \leq\left\|y_{n}-x_{n}\right\|^{2}+2\left\|y_{n}-x_{n}\right\|\left\|x_{n}-y_{n-1}\right\|+\left\|x_{n}-y_{n-1}\right\|^{2} \\
& \leq\left\|y_{n}-x_{n}\right\|^{2}+(\sqrt{2}+1)\left\|y_{n}-x_{n}\right\|^{2}+\frac{1}{\sqrt{2}+1}\left\|x_{n}-y_{n-1}\right\|^{2}+\left\|x_{n}-y_{n-1}\right\|^{2} \\
& =(\sqrt{2}+2)\left\|y_{n}-x_{n}\right\|^{2}+\sqrt{2}\left\|x_{n}-y_{n-1}\right\|^{2} .
\end{aligned}
$$

Substituting (3.14) into (3.13) and using Lemma 2.13, we have

$$
\begin{aligned}
& 2 \lambda_{n}\left\langle x_{n+1}-y_{n}, A y_{n-1}-A y_{n}\right\rangle \\
& \leq \mu \frac{\lambda_{n}}{\lambda_{n+1}}\left(\sqrt{2}\left\|x_{n+1}-y_{n}\right\|^{2}+(\sqrt{2}+1)\left\|y_{n}-x_{n}\right\|^{2}+\left\|x_{n}-y_{n-1}\right\|^{2}\right) \\
& \leq \mu \frac{\lambda_{n}}{\lambda_{n+1}}\left(\delta \sqrt{2} \phi\left(x_{n+1}, y_{n}\right)+\delta(\sqrt{2}+1) \phi\left(y_{n}, x_{n}\right)+\delta \phi\left(x_{n}, y_{n-1}\right)\right) \\
& =\theta_{n}\left(\sqrt{2} \phi\left(x_{n+1}, y_{n}\right)+(\sqrt{2}+1) \phi\left(y_{n}, x_{n}\right)+\phi\left(x_{n}, y_{n-1}\right)\right),
\end{aligned}
$$

where $\theta_{n}=\frac{\mu \delta \lambda_{n}}{\lambda_{n+1}}$. Substituting (3.15) into (3.12), we have

$$
\begin{aligned}
\phi\left(p, x_{n+1}\right) \leq & \phi\left(p, x_{n}\right)-\phi\left(x_{n+1}, y_{n}\right)-\phi\left(y_{n}, x_{n}\right) \\
& +\theta_{n}\left(\sqrt{2} \phi\left(x_{n+1}, y_{n}\right)+(\sqrt{2}+1) \phi\left(y_{n}, x_{n}\right)+\phi\left(x_{n}, y_{n-1}\right)\right) \\
= & \phi\left(p, x_{n}\right)+\theta_{n} \phi\left(x_{n}, y_{n-1}\right)-\left(1-(\sqrt{2}+1) \theta_{n}\right) \phi\left(y_{n}, x_{n}\right)-\left(1-\sqrt{2} \theta_{n}\right) \phi\left(x_{n+1}, y_{n}\right) .
\end{aligned}
$$

Adding the term $\theta_{n+1} \phi\left(x_{n+1}, y_{n}\right)$ to both sides of (3.16), we get

$$
\begin{aligned}
\phi\left(p, x_{n+1}\right)+\theta_{n+1} \phi\left(x_{n+1}, y_{n}\right) \leq & \phi\left(p, x_{n}\right)+\theta_{n} \phi\left(x_{n}, y_{n-1}\right)-\left(1-(\sqrt{2}+1) \theta_{n}\right) \phi\left(y_{n}, x_{n}\right) \\
& -\left(1-\sqrt{2} \theta_{n}-\theta_{n+1}\right) \phi\left(x_{n+1}, y_{n}\right) .
\end{aligned}
$$

Then (3.17) reduces to the following inequality:

$$
\mathscr{A}_{n+1} \leq \mathscr{A}_{n}-\left(1-(\sqrt{2}+1) \theta_{n}\right) \phi\left(y_{n}, x_{n}\right)-\left(1-\sqrt{2} \theta_{n}-\theta_{n+1}\right) \phi\left(x_{n+1}, y_{n}\right),
$$

where $\mathscr{A}_{n}=\phi\left(p, x_{n}\right)+\theta_{n} \phi\left(x_{n}, y_{n-1}\right)$.

Lemma 3.4. Assume that Assumptions $(A 1)-(A 4)$ hold. Let $\left\{x_{n}\right\}$ be a sequence generated by Algorithm 1. Suppose that there exists a subsequence $\left\{x_{n_{k}}\right\}$ of $\left\{x_{n}\right\}$ such that $\left\{x_{n_{k}}\right\}$ converges weakly to $z \in E$. If $\lim _{n \rightarrow \infty}\left\|x_{n}-y_{n}\right\|=0$ and $\lim _{n \rightarrow \infty}\left\|y_{n+1}-y_{n}\right\|=0$, then $z \in \operatorname{VI}(C, A)$.

Proof. Let $\left\{x_{n_{k}}\right\}$ be the subsequence of $\left\{x_{n}\right\}$ such that $x_{n_{k}} \rightarrow z \in E$. Since $\lim _{n \rightarrow \infty}\left\|x_{n}-y_{n}\right\|=0$ and $\left\{y_{n}\right\} \subset C$, we have $y_{n_{k}} \rightarrow z$ and $z \in C$. From the definition of $y_{n_{k}}$, we see that

$$
y_{n_{k}+1}=\Pi_{C} J^{-1}\left(J x_{n_{k}+1}-\lambda_{n_{k}+1} A y_{n_{k}}\right) .
$$

By Lemma $2.12(i)$, we have

$$
\left\langle x-y_{n_{k}+1}, J y_{n_{k}+1}-J x_{n_{k}+1}+\lambda_{n_{k}+1} A y_{n_{k}}\right\rangle \geq 0, \forall x \in C,
$$

which implies that

$$
\lambda_{n_{k}+1}\left\langle x-y_{n_{k}+1}, A y_{n_{k}}\right\rangle \geq\left\langle x-y_{n_{k}+1}, J x_{n_{k}+1}-J y_{n_{k}+1}\right\rangle, \forall x \in C .
$$


Moreover, we have

$$
\left\langle x-y_{n_{k}}, A y_{n_{k}}\right\rangle \geq \frac{1}{\lambda_{n_{k}}}\left\langle x-y_{n_{k}+1}, J x_{n_{k}+1}-J y_{n_{k}+1}\right\rangle+\left\langle y_{n_{k}+1}-y_{n_{k}}, A y_{n_{k}}\right\rangle .
$$

Since $\lim _{k \rightarrow \infty} \lambda_{n_{k}}=\lambda>0,\left\{A y_{n_{k}}\right\}$ is bounded and $J$ is norm-to-norm uniform continuous, we have

$$
\liminf _{k \rightarrow \infty}\left\langle x-y_{n_{k}}, A y_{n_{k}}\right\rangle \geq 0 .
$$

Let $\left\{\varepsilon_{k}\right\}$ be a decreasing sequence of positive real numbers such that $\varepsilon_{k} \rightarrow 0$ as $k \rightarrow \infty$. For each $\varepsilon_{k}$, we denote by $N$ the smallest positive integer such that

$$
\left\langle x-y_{n_{k}}, A y_{n_{k}}\right\rangle+\varepsilon_{k} \geq 0, \forall k \geq N .
$$

It is clear that (3.21) can be written as

$$
\left\langle x+\varepsilon_{k} v_{n_{k}}-y_{n_{k}}, A y_{n_{k}}\right\rangle \geq 0, \forall k \geq N
$$

for some $v_{n_{k}}:=w \in E$ satisfying $\left\langle v_{n_{k}}, A y_{n_{k}}\right\rangle=1$ (since $A y_{n_{k}} \neq 0$ ). By the pseudo-monotonicity of $A$, we have

$$
\left\langle x+\varepsilon_{k} v_{n_{k}}-y_{n_{k}}, A\left(x+\varepsilon_{k} v_{n_{k}}\right)\right\rangle \geq 0 .
$$

Since $y_{n_{k}} \rightarrow z, \varepsilon_{k} \rightarrow 0$ and $A$ is Lipschitz continuous (hence it is continuous), it follows from (3.23) that

$$
\langle x-z, A x\rangle \geq 0, \forall x \in C .
$$

By Lemma $2.14(i i)$, we obtain $z \in V I(C, A)$.

Theorem 3.5. Assume that Assumptions $(A 1)-(A 4)$ hold. Let $\left\{x_{n}\right\}$ be a sequence generated by Algorithm 1. Then $\left\{x_{n}\right\}$ converges weakly to an element in $\operatorname{VI}(C, A)$.

Proof. Since $\lim _{n \rightarrow \infty} \lambda_{n}$ exists and $\mu \in\left(0, \frac{\sqrt{2}-1}{\delta}\right)$, we have

$$
\lim _{n \rightarrow \infty}\left(1-(\sqrt{2}+1) \theta_{n}\right)=\lim _{n \rightarrow \infty}\left(1-\sqrt{2} \theta_{n}-\theta_{n+1}\right)=1-(\sqrt{2}+1) \mu \delta=1-\frac{\mu \delta}{\sqrt{2}-1}>0 .
$$

Thus there exists $n_{0} \in \mathbb{N}$ such that

$$
1-(\sqrt{2}+1) \theta_{n}>0 \text { and } 1-\sqrt{2} \theta_{n}-\theta_{n+1}>0, \forall n \geq n_{0},
$$

which implies that

$$
\left(1-(\sqrt{2}+1) \theta_{n}\right) \phi\left(y_{n}, x_{n}\right)+\left(1-\sqrt{2} \theta_{n}-\theta_{n+1}\right) \phi\left(x_{n+1}, y_{n}\right) \geq 0, \forall n \geq n_{0} .
$$

Now, we can write (3.18) in the following form:

$$
\mathscr{A}_{n+1} \leq \mathscr{A}_{n}-\mathscr{B}_{n}, \forall n \geq n_{0},
$$

where $\mathscr{B}_{n}=\left(1-(\sqrt{2}+1) \theta_{n}\right) \phi\left(y_{n}, x_{n}\right)+\left(1-\sqrt{2} \theta_{n}-\theta_{n+1}\right) \phi\left(x_{n+1}, y_{n}\right)$. By Lemma 2.16, we obtain $\lim _{n \rightarrow \infty} \mathscr{A}_{n}$ exists and

$$
\sum_{n=n_{0}}^{\infty} \mathscr{B}_{n}=\sum_{n=n_{0}}^{\infty}\left[\left(1-(\sqrt{2}+1) \theta_{n}\right) \phi\left(y_{n}, x_{n}\right)+\left(1-\sqrt{2} \theta_{n}-\theta_{n+1}\right) \phi\left(x_{n+1}, y_{n}\right)\right]<\infty .
$$

Thus we have $\lim _{n \rightarrow \infty} \phi\left(y_{n}, x_{n}\right)=\lim _{n \rightarrow \infty} \phi\left(x_{n+1}, y_{n}\right)=0$. Using Lemma 2.13, we get

$$
\lim _{n \rightarrow \infty}\left\|y_{n}-x_{n}\right\|=\lim _{n \rightarrow \infty}\left\|x_{n+1}-y_{n}\right\|=0 \text {. }
$$


By the uniform continuity of $J$, we have

$$
\lim _{n \rightarrow \infty}\left\|J y_{n}-J x_{n}\right\|=\lim _{n \rightarrow \infty}\left\|J x_{n+1}-J y_{n}\right\|=0,
$$

which implies that

$$
\left\|J y_{n}-J y_{n-1}\right\| \leq\left\|J y_{n}-J x_{n}\right\|+\left\|J x_{n}-J y_{n-1}\right\| \rightarrow 0 .
$$

Also, by the norm to norm uniform continuity of $J^{-1}$, we have

$$
\lim _{n \rightarrow \infty}\left\|y_{n}-y_{n-1}\right\|=0 \text {. }
$$

Since $\lim _{n \rightarrow \infty} \mathscr{A}_{n}$ exists and from (3.25), we have that $\left\{\phi\left(p, x_{n}\right)\right\}$ is bounded. It follows from Lemma 2.13 that $\left\{x_{n}\right\}$ is bounded. By the reflexivity of $E$, there exists a subsequence $\left\{x_{n_{k}}\right\}$ of $\left\{x_{n}\right\}$ such that $x_{n_{k}} \rightarrow z \in E$. As proved in Lemma 3.4, we have $z \in V I(C, A)$. In summary, we have shown that

(i) $\lim _{n \rightarrow \infty} \phi\left(p, x_{n}\right)$ exists for every $p \in V I(C, A)$;

(ii) every sequential weak limit point of $\left\{x_{n}\right\}$ is in $\operatorname{VI}(C, A)$.

Therefore, by Lemma 2.17, we conclude that $\left\{x_{n}\right\}$ converges weakly to an element in $V I(C, A)$. This completes the proof.

\section{NUMERICAL EXPERIMENTS}

In this section, we provide several numerical experiments to illustrate the convergence and the efficiency of our Algorithm 1 in solving the variational inequality problem. Moreover, we also compare it with subgradient extragradient method (SEM) (1.5), Popov's subgradient extragradient method (PSEM) (1.6), Halpern's subgradient extragradient method (HSEM) proposed in ([11, Algorithm 3.5]) and modified subgradient extragradient method with an Armijo line search rule (L-MSEM) proposed in ([31, Algorithm 3.1]). We have used the following values of the control parameters for the whole numerical part:

(i) subgradient extragradient method (SEM):

$$
\lambda=\frac{0.7}{L}, D_{n}=\left\|x_{n+1}-x_{n}\right\|
$$

(ii) Popov's subgradient extragradient method (PSEM):

$$
\lambda=\frac{0.7}{3 L}, x_{0}=y_{0}, D_{n}=\left\|x_{n+1}-x_{n}\right\|
$$

(iii) Halpern's subgradient extragradient method (HSEM):

$$
\lambda=\frac{0.7}{L}, \alpha_{n}=\frac{1}{n+2}, D_{n}=\left\|x_{n+1}-x_{n}\right\|
$$

(iv) modified subgradient extragradient method with an Armijo line search rule (L-MSEM):

$$
\mu=0.80, \gamma=1, l=0.20, D_{n}=\left\|x_{n+1}-x_{n}\right\| ;
$$

(v) Algorithm 1 (modified Popov's subgradient extragradient method (M-PSEM)):

$$
\mu=0.33, \lambda_{0}=\lambda_{1}=0.20, x_{0}=y_{0}, D_{n}=\left\|x_{n+1}-x_{n}\right\| .
$$


Example 4.1. We consider the HpHard problem which is taken from [32]. Let $A: \mathbb{R}^{m} \rightarrow \mathbb{R}^{m}$ be an operator defined by $A x=M x+q$ with $q \in \mathbb{R}^{m}$ and

$$
M=N N^{T}+B+D,
$$

where $N$ is an $m \times m$ matrix, $B$ is an $m \times m$ skew-symmetric matrix and $D$ is an $m \times m$ positive definite diagonal matrix. The feasible set is

$$
C=\left\{x \in \mathbb{R}^{m}: Q x \leq b\right\},
$$

where $Q$ is an $100 \times m$ matrix and $b$ is a nonnegative vector in $\mathbb{R}^{m}$. It is clear that $A$ is monotone and Lipschitz continuous with $L=\|M\|$ (hence the variational inequality has a unique solution). For $q=0$, the solution set of the corresponding variational inequality is $\operatorname{VI}(C, A)=\{0\}$. We perform numerical experiments with the starting point $x_{0}=(1,1, \ldots, 1)^{T}$ and use $\left\|x_{n+1}-x_{n}\right\|<$ $\varepsilon=10^{-3}$ to stop the iterative process. The numerical results of all methods have been reported in the Figures 1-3.
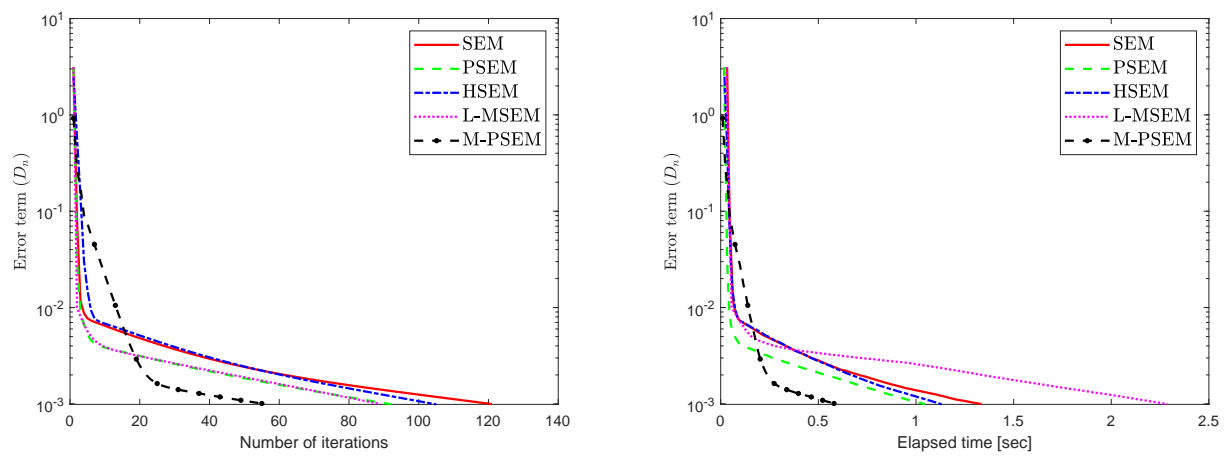

FIGURE 1. Numerical comparison of M-PSEM with existing algorithms when $m=10$ for Example 4.1.
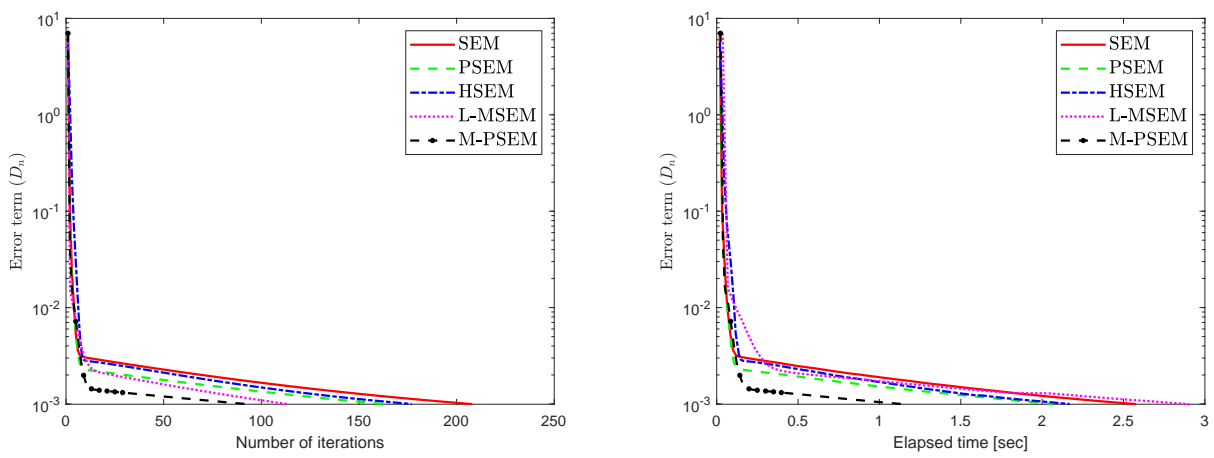

FIGURE 2. Numerical comparison of M-PSEM with existing algorithms when $m=50$ for Example 4.1.

Example 4.2. In this example, we take $E=L_{2}([0,1])$ with the norm

$$
\|x\|_{2}=\left(\int_{0}^{1}|x(t)|^{2} d t\right)^{1 / 2}
$$



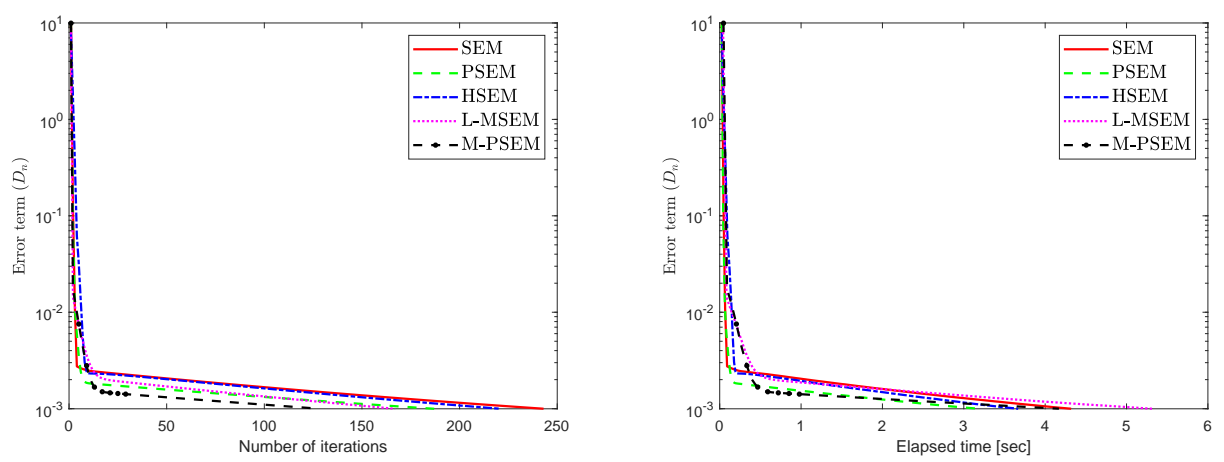

FIGURE 3. Numerical comparison of M-PSEM with existing algorithms when $m=100$ for Example 4.1.

and the inner product

$$
\langle x, y\rangle=\int_{0}^{1} x(t) y(t) d t
$$

for all $x, y \in L_{2}([0,1])$. The feasible set is $C=\{x \in E:\|x\| \leq 1\}$. Define an integral operator $A: C \rightarrow E$ by

$$
A x(t)=\int_{0}^{1}(x(t)-f(t, s) g(x(s))) d s-h(t), x \in C \text { and } t \in[0,1],
$$

where

$$
f(t, s)=\frac{2 t s e^{t+s}}{e \sqrt{e^{2}-1}}, g(x)=\cos x \text { and } h(t)=\frac{2 t e^{t}}{e \sqrt{e^{2}-1}} .
$$

It was shown that $A$ is monotone (hence it is pseudo-monotone) and Lipschitz continuous with $L=2$ (see [33]). The solution set of the corresponding variational inequality problem is $V I(C, A)=\{0\}$. We perform numerical experiments with three different starting points and use $\left\|x_{n+1}-x_{n}\right\|<\varepsilon=10^{-4}$ to stop the iterative process. The numerical results of all methods have been reported in the Figures 4-6.
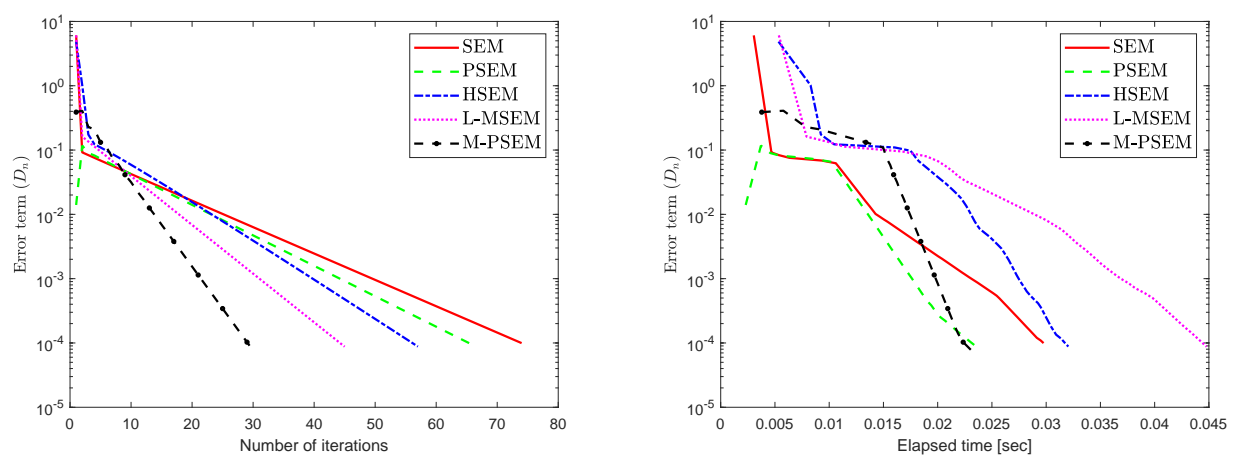

FIGURE 4. Numerical comparison of M-PSEM with existing algorithms when $x_{0}=1$ for Example 4.2. 

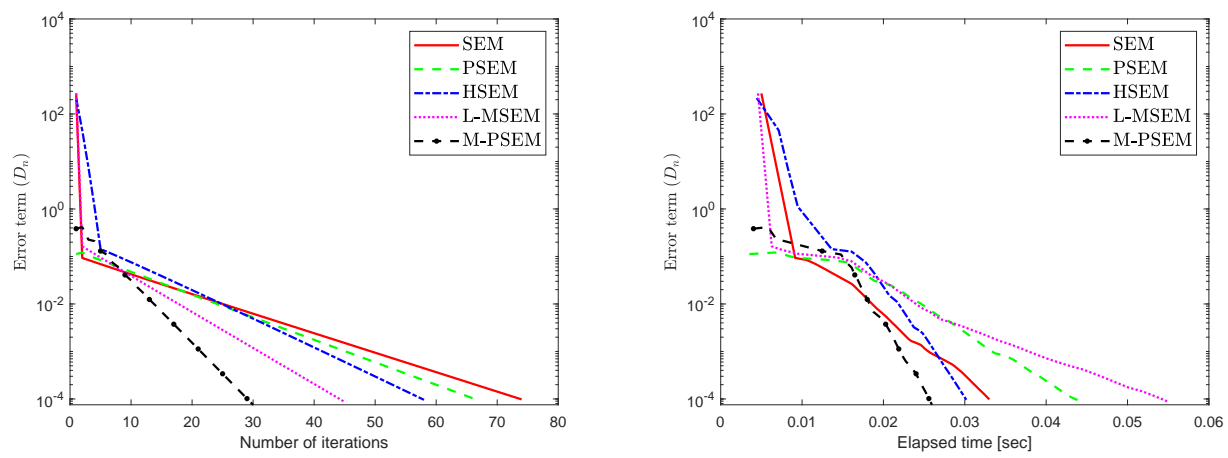

FIGURE 5. Numerical comparison of M-PSEM with existing algorithms when $x_{0}=t$ for Example 4.2.
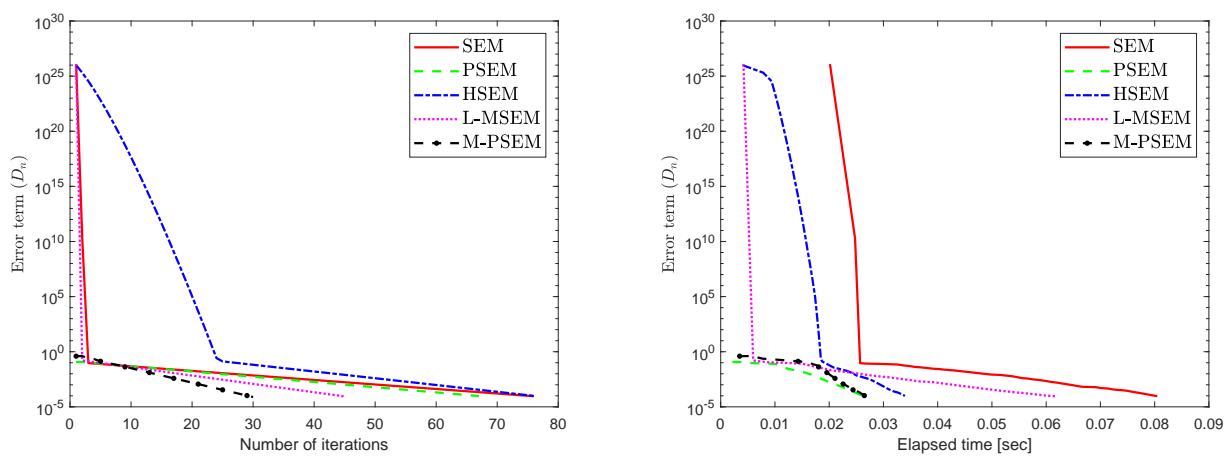

FIGURE 6. Numerical comparison of M-PSEM with existing algorithms when $x_{0}=e^{t}$ for Example 4.2.

Example 4.3. Consider the following pseudo-monotone variational inequalities with

$$
A x=\left(\begin{array}{l}
x_{1}+x_{2}+x_{3}+x_{4}-4 x_{2} x_{3} x_{4} \\
x_{1}+x_{2}+x_{3}+x_{4}-4 x_{1} x_{3} x_{4} \\
x_{1}+x_{2}+x_{3}+x_{4}-4 x_{1} x_{2} x_{4} \\
x_{1}+x_{2}+x_{3}+x_{4}-4 x_{1} x_{2} x_{3}
\end{array}\right) .
$$

The feasible set is $C=\left\{x \in \mathbb{R}^{4}: 1 \leq x_{1} \leq 5, i=1,2,3,4\right\}$. It is easy to see that $A x$ is not monotone on $C$. Using the Monte Carlo approach [34], it can be shown that $A$ is pseudomonotone on $C$. This problem has unique solution $z=(5,5,5,5)^{T}$. Since the Lipschitz constant is unknown, thus SEM, PSEM and HSEM do not applicable in this problem. We only perform the numerical experiments of L-PSEM and M-MSEM with three different starting points $x_{0}$ and use $\left\|x_{n+1}-x_{n}\right\|<\varepsilon$ to stop the iterative process. The numerical results of mentioned methods have been reported in the Tables 1 and 2 .

In the last example, we use our proposed algorithm to solve the pseudo-convex minimization problem in a finite dimension space $E=\mathbb{R}^{m}$. Let $C$ be a nonempty, closed and convex subset of $E$. Recall that a differentiable function $f: E \rightarrow \mathbb{R}$ is called pseudo-convex if

$$
\langle\nabla f(x), y-x\rangle \geq 0 \Longrightarrow f(y) \geq f(x), \forall x, y \in C,
$$


TABLE 1. Numerical tests of L-PSEM with different starting points for Example 4.3.

\begin{tabular}{lcccccccc}
\hline$\varepsilon$ & 0.01 & 0.001 & 0.0001 & 0.00001 & 0.01 & 0.001 & 0.0001 & 0.00001 \\
$x_{0}$ & No. of Iter. & No. of Iter. & No. of Iter. & No. of Iter. & Time(s) & Time(s) & Time(s) & Time(s) \\
\hline \hline$(-2,2,8,10)^{T}$ & 3 & 3 & 3 & 3 & 0.0633 & 0.0701 & 0.0865 & 0.0723 \\
$(-1,2,2,5)^{T}$ & 3 & 3 & 3 & 3 & 0.0723 & 0.0721 & 0.0789 & 0.0768 \\
$(3,1,-5,-2)^{T}$ & 3 & 3 & 3 & 3 & 0.0770 & 0.0821 & 0.0871 & 0.0799 \\
\hline \hline
\end{tabular}

TABLE 2. Numerical tests of M-PSEM with different starting points for Example 4.3.

\begin{tabular}{lcccccccc}
\hline$\varepsilon$ & 0.01 & 0.001 & 0.0001 & 0.00001 & 0.01 & 0.001 & 0.0001 & 0.00001 \\
$x_{0}$ & No. of Iter. & No. of Iter. & No. of Iter. & No. of Iter. & Time(s) & Time(s) & Time(s) & Time(s) \\
\hline \hline$(-2,2,8,10)^{T}$ & 5 & 5 & 5 & 5 & 0.0298 & 0.0234 & 0.0395 & 0.0415 \\
$(-1,2,2,5)^{T}$ & 5 & 5 & 5 & 5 & 0.0394 & 0.0440 & 0.0387 & 0.0382 \\
$(3,1,-5,-2)^{T}$ & 4 & 4 & 4 & 4 & 0.0806 & 0.0264 & 0.0271 & 0.0269 \\
\hline \hline
\end{tabular}

where $\nabla f$ is the gradient of $f$. The pseudo-convex minimization problem is to find an element $z \in C$ such that

$$
f(z)=\min _{x \in C} f(x),
$$

where $f$ is differentiable and pseudo-convex. This problem (4.1) is equivalent to the following variational inequality problem $[35,36]$ :

$$
\langle\nabla f(z), x-z\rangle \geq 0, \forall x \in C .
$$

It is known that a differentiable function is pseudo-convex if and only if its gradient is a pseudomonotone mapping (see [37]).

Example 4.4. Consider the quadratic fractional programming problem in the following form [34]:

$$
\left\{\begin{array}{l}
\min f(x)=\frac{x^{T} Q x+a^{T} x+a_{0}}{b^{T} x+b_{0}}, \\
\text { subject to } x \in K=\left\{x \in \mathbb{R}^{4}: b^{T} x+b_{0}>0\right\}
\end{array}\right.
$$

where

$$
Q=\left(\begin{array}{cccc}
5 & -1 & 2 & 0 \\
-1 & 5 & -1 & 3 \\
2 & -1 & 3 & 0 \\
0 & 3 & 0 & 5
\end{array}\right), a=\left(\begin{array}{c}
1 \\
-2 \\
-2 \\
1
\end{array}\right), b=\left(\begin{array}{l}
2 \\
1 \\
1 \\
0
\end{array}\right), a_{0}=-2 \text { and } b_{0}=4
$$

It is easy to verify that $Q$ is symmetric and positive definite on $\mathbb{R}^{4}$ and consequently $f$ is pseudoconvex on $K$. Hence $\nabla f$ is pseudo-monotone. Using the quotient rule, we get

$$
\nabla f(x)=\frac{\left(b^{T} x+b_{0}\right)(2 Q x+a)-b\left(x^{T} Q+a^{T} x+a_{0}\right)}{\left(b^{T} x+b_{0}\right)^{2}} .
$$

In this point of view, we can set $A=\nabla f$ in Theorem 3.5. We minimize $f$ over $C=\left\{x \in \mathbb{R}^{4}: 1 \leq\right.$ $\left.x_{i} \leq 10, i=1,2,3,4\right\} \subset K$. This problem has a unique solution $z=(1,1,1,1)^{T} \in C$. Since the Lipschitz constant is unknown, thus SEM, PSEM and HSEM do not applicable in this problem. 
We only perform the numerical experiments of L-PSEM and M-MSEM with three different starting points $x_{0}$ and use $\left\|x_{n+1}-x_{n}\right\|<\varepsilon$ to stop the iterative process. The numerical results of mentioned methods have been reported in the Tables 3 and 4.

TABLE 3. Numerical tests of L-PSEM with different starting points for Example 4.4.

\begin{tabular}{lcccccccc}
\hline$\varepsilon$ & 0.01 & 0.001 & 0.0001 & 0.00001 & 0.01 & 0.001 & 0.0001 & 0.00001 \\
$x_{0}$ & No. of Iter. & No. of Iter. & No. of Iter. & No. of Iter. & Time(s) & Time(s) & Time(s) & Time(s) \\
\hline \hline$(10,10,10,10)^{T}$ & 58 & 101 & 141 & 187 & 0.6008 & 1.4598 & 1.9081 & 3.7862 \\
$(10,20,30,40)^{T}$ & 73 & 113 & 156 & 233 & 0.9732 & 1.7781 & 2.2009 & 4.5341 \\
$(20,-20,20,-20)^{T}$ & 81 & 141 & 199 & 243 & 1.1023 & 1.8970 & 4.0032 & 4.9032 \\
\hline \hline
\end{tabular}

TABLE 4. Numerical tests of M-PSEM with different starting points for Example 4.4.

\begin{tabular}{lcccccccc}
\hline$\varepsilon$ & 0.01 & 0.001 & 0.0001 & 0.00001 & 0.01 & 0.001 & 0.0001 & 0.00001 \\
$x_{0}$ & No. of Iter. & No. of Iter. & No. of Iter. & No. of Iter. & Time(s) & Time(s) & Time(s) & Time(s) \\
\hline \hline$(10,10,10,10)^{T}$ & 49 & 91 & 133 & 175 & 0.2203 & 0.4282 & 0.7989 & 0.8634 \\
$(10,20,30,40)^{T}$ & 69 & 102 & 148 & 191 & 0.2197 & 0.6281 & 1.2019 & 1.3451 \\
$(20,-20,20,-20)^{T}$ & 87 & 158 & 201 & 295 & 0.5214 & 1.3122 & 1.9514 & 2.4151 \\
\hline \hline
\end{tabular}

\section{CONCLUSION}

In recent years, several variants of the Popov's subgradient extragradient method have been studied intensively by many authors. Note that most of them were studied in Hilbert spaces. In this paper, we extend the Popov's subgradient extragradient method to Banach spaces. The weak convergence theorem of the proposed algorithm was proved without the knowledge of the Lipschitz constant of the mapping. Several numerical experiments are performed to illustrate the performance of our algorithm.

\section{Funding}

The first author was supported by RMUTT Research Grant for New Scholar under Grant NSF62D0602. The second author was supported by the Petchra Pra Jom Klao Doctoral Scholarship Academic for Ph.D. Program at KMUTT (Grant No. 39/2560).

\section{REFERENCES}

[1] V. Barbu, T. Precupanu, Convexity and Optimization in Banach Spaces, Springer Dordrecht Heidelberg London, New York, 2010.

[2] D.P. Bertsekas, E.M. Gafni, Projection methods for variational inequalities with applications to the traffic assignment problem, Math. Prog. Study 17 (1982), 139-159.

[3] S. Dafermos, Exchange price equilibria and variational inequalities, Math. Programming 46 (1990), $391-402$.

[4] N. Kikuchi, J.T. Oden, Contact Problems in Elasticity, SIAM, Philadelphia, PA, 1988.

[5] T. Humphries, M. Loreto, B. Halter, W. O’Keeffe, L. Ramirez, Comparison of regularized and superiorized methods for tomographic image reconstruction, J. Appl. Numer. Optim. 2 (2020), 77-99.

[6] N.T. An, Solving k-center problems involving sets based on optimization techniques, J. Global Optim. 76 (2020), 189-209. 
[7] E. Bonacker, A. Gibali, Karl-Heinz Küfer, Nesterov perturbations and projection methods applied to IMRT, J. Nonlinear Var. Anal. 4 (2020), 63-86.

[8] B.S. He, L.Z. Liao, Improvements of some projection methods for monotone nonlinear variational inequalities, J. Optim. Theory Appl. 112 (2002), 111-128.

[9] G.M. Korpelevich, The extragradient method for finding saddle points and other problems, Matecon 12 (1976), 747-756.

[10] J. Mashreghi, M. Nasri, Forcing strong convergence of Korpelevich's method in Banach spaces with its applications in game theory, Nonlinear Anal. 72 (2010), 2086-2099.

[11] G. Cai, A. Gibali, O.S. Iyiola, Y. Shehu, A New Double-Projection Method for Solving Variational Inequalities in Banach Spaces, J Optim Theory Appl 178 (2018), 219-239.

[12] S.Y. Cho, A monotone Bregan projection algorithm for fixed point and equilibrium problems in a reflexive Banach space, Filomat, 34 (2020), 1487-1497.

[13] L.C. Ceng, Q.H. Ansari, J.C. Yao Relaxed extragradient methods for finding minimum-norm solutions of the split feasibility problem, Nonlinear Anal. 75 (2012), 2116-2125.

[14] Y. Shehu, O.S. Iyiola, Strong convergence result for monotone variational inequalities, Numer. Algo. 76 (2017), 259-282.

[15] D.V. Thong, D.V. Hieu, New extragradient methods for solving variational inequality problems and fixed point problems, J. Fixed Point Theory Appl. 20 (2018), 129.

[16] D.V. Thong and D.V. Hieu, Modified subgradient extragradient method for variational inequality problems. Numer. Algo. 79 (2018), 597-610.

[17] L.D. Popov, A modification of the Arrow-Hurwicz method for searching for saddle points, Mat. Zametki, 28 (1980), 777-784.

[18] Y. Censor, A. Gibali, S. Reich, The subgradient extragradient method for solving variational inequalities in Hilbert space, J Optim Theory Appl.148 (2011), 318-335.

[19] Y.V. Malitsky, V.V. Semenov, An extragradient algorithm for monotone variational inequalities, Cybern. Syst. Anal. 50 (2014), 271-277.

[20] A. Gibali, D.V. Thong, A new low-cost double projection method for solving variational inequalities, Optim. Eng. 21 (2020), 1613-1634.

[21] H. ur Rehman, P. Kumam, Q.L. Dong, Y. Peng and W. Deebani, A new Popov's subgradient extragradient method for two classes of equilibrium programming in a real Hilbert space, Optimization, DOI: 10.1080/02331934.2020.1797026.

[22] H. ur Rehman, P. Kumam, Y.J. Cho, Y.I. Suleiman, W. Kumam, Modified Popov's explicit iterative algorithms for solving pseudomonotone equilibrium problems, Optim. Methods Softw. 36 (2021), 82-113.

[23] I. Cioranescu, Geometry of Banach Spaces, Duality Mappings and Nonlinear Problems, Kluwer Academic, Dordrecht, 1990.

[24] S. Reich, Review of geometry of Banach spaces, in: Duality Mappings and Nonlinear Problems by loana Cioranescu, Kluwer Academic Publishers, Dordrecht, 1990.

[25] W. Takahashi, Nonlinear Functional Analysis, Yokohama Publishers, Yokohama, 2000.

[26] H.K. Xu, Inequalities in Banach spaces with applications, Nonlinear Anal. 16 (1991), 1127-1138.

[27] P.D. Khanh, P.T. Vuong, Modified projection method for strongly pseudo-monotone variational inequalities, J. Global Optim. 58 (2014), 341-350.

[28] Y.I. Alber, Metric and generalized projection operators in Banach spaces: properties and applications, in: A.G. Kartsatos (Ed.), Theory and Applications of Nonlinear Operator of Accretive and Monotone Type, pp. 15-50, Marcel Dekker, New York, 1996,

[29] K. Aoyama and F. Kohsaka, Strongly relatively nonexpansive sequences generated by firmly nonexpansivelike mappings, Fixed Point Theory Appl 2014 (2014), 95.

[30] R. E. Showalter, Monotone Operators in Banach Space and Nonlinear Partial Differential Equations, Mathematical Surveys and Monographs, Vol. 49, 1997

[31] P.Q. Khanh, D.V. Thong, N.T. Vinh, Versions of the Subgradient Extragradient Method for Pseudomonotone Variational Inequalities, Acta Appl Math (2020). https://doi.org/10.1007/s10440-020-00335-9. 
[32] P.T. Harker, J.S. Pang, A damped-Newton method for the linear complementarity problem. In: Allgower, G., Georg, K. (eds.) Computational Solution of Nonlinear Systems of Equations. Lectures in Applied Mathematics, vol. 26, pp. 265-284, AMS, Providence, 1990.

[33] D.V. Hieu, P.K. Anh, L.D. Muu, Modified hybrid projection methods for finding common solutions to variational inequality problems, Comput. Optim. Appl. 66 (2017), 75-96.

[34] X. Hu, J. Wang, Solving pseudo-monotone variational inequalities and pseudo-convex optimization problems using the projection neural network, IEEE Trans. Neural. Netw. 17 (2006), 1487-1499.

[35] D. Kinderlehrer, G. Stampacchia, An Introduction to Variational Inequalities and Their Applications, Academic Press, New York, 1980.

[36] P.T. Harker, J.S. Pang, Finite-dimensional variational inequality and nonlinear complementarity problems: a survey of theory, algorithms and applications. Math Program. 48 (1990), 161-220.

[37] S. Karamardian, S. Schaible, Seven kinds of monotone maps, J Optim Theory Appl. 66 (1990), 37-46. 